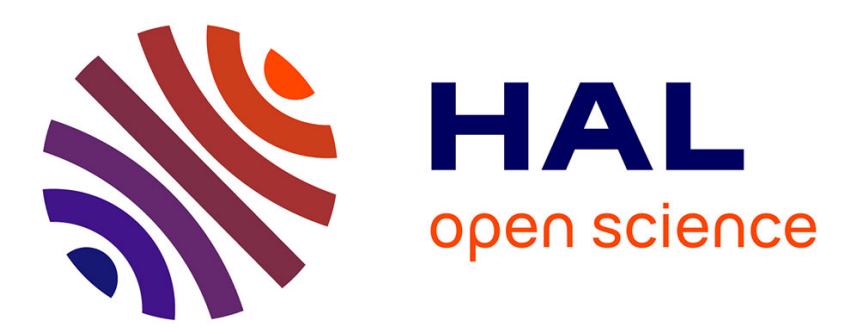

\title{
Nanoscale spintronic oscillators based on the excitation of confined soliton modes
}

G. Finocchio, V. Puliafito, S. Komineas, L. Torres, O. Ozatay, Thomas Hauet, B. Azzerboni

\section{- To cite this version:}

G. Finocchio, V. Puliafito, S. Komineas, L. Torres, O. Ozatay, et al.. Nanoscale spintronic oscillators based on the excitation of confined soliton modes. Journal of Applied Physics, 2013, 114, pp.163908. 10.1063/1.4827384 . hal-01276625

\section{HAL Id: hal-01276625 \\ https://hal.science/hal-01276625}

Submitted on 13 Jul 2016

HAL is a multi-disciplinary open access archive for the deposit and dissemination of scientific research documents, whether they are published or not. The documents may come from teaching and research institutions in France or abroad, or from public or private research centers.
L'archive ouverte pluridisciplinaire HAL, est destinée au dépôt et à la diffusion de documents scientifiques de niveau recherche, publiés ou non, émanant des établissements d'enseignement et de recherche français ou étrangers, des laboratoires publics ou privés. 


\section{AIP Appilited Physics}

Nanoscale spintronic oscillators based on the excitation of confined soliton modes

G. Finocchio, V. Puliafito, S. Komineas, L. Torres, O. Ozatay et al.

Citation: J. Appl. Phys. 114, 163908 (2013); doi: 10.1063/1.4827384

View online: http://dx.doi.org/10.1063/1.4827384

View Table of Contents: http://jap.aip.org/resource/1/JAPIAU/v114/i16

Published by the AIP Publishing LLC.

Additional information on J. Appl. Phys.

Journal Homepage: http://jap.aip.org/

Journal Information: http://jap.aip.org/about/about_the_journal

Top downloads: http://jap.aip.org/features/most_downloaded

Information for Authors: http://jap.aip.org/authors

\section{AlP Re-register for Table of Content Alerts}

\section{Create a profile. \\ Sign up today!}




\title{
Nanoscale spintronic oscillators based on the excitation of confined soliton modes
}

\author{
G. Finocchio, ${ }^{1, a)}$ V. Puliafito, ${ }^{1}$ S. Komineas, ${ }^{2}$ L. Torres, ${ }^{3}$ O. Ozatay, ${ }^{4}$ T. Hauet, ${ }^{5}$ \\ and B. Azzerboni ${ }^{1}$ \\ ${ }^{1}$ Department of Electronic Engineering, Industrial Chemistry and Engineering, University of Messina, \\ Messina, Italy \\ ${ }^{2}$ Department of Applied Mathematics, University of Crete, Heraklion, Greece \\ ${ }^{3}$ Department of Applied Physics, University of Salamanca, Salamanca, Spain \\ ${ }^{4}$ Department of Physics, Bogazici University, Istanbul, Turkey \\ ${ }^{5}$ Institut Jean Lamour, Université de Lorraine-CNRS UMR 7198, Nancy, France
}

(Received 18 July 2013; accepted 14 October 2013; published online 29 October 2013)

\begin{abstract}
This paper demonstrates how to excite complex soliton modes in nanomagnets with perpendicular to plane magnetic anisotropy driven by the non-uniform injection of a spin-polarized current. We addressed the study toward two different scenarios, in the first the excitation of two rotating bubble/antibubble pairs is predicted, in the second one, by means of the topological density, we characterized the dissipative droplet recently measured as single constrained bubble/antibubble pair. Our results are important for the theoretical understanding of how to control the spatial structure of soliton modes for application in spintronics, magnonics, and domain wall devices. (C) 2013 AIP Publishing LLC. [http://dx.doi.org/10.1063/1.4827384]
\end{abstract}

\section{INTRODUCTION}

Nonlinear magnetization dynamics of magnetic solitons exhibiting persistent magnetization motion show original features of high interest from a fundamental and technological point of view. ${ }^{1,2}$ Most of the theoretical studies of the soliton dynamics are based on the solution of Landau-LifshitzGilbert equation in its conservative form, or on the analysis of the relaxation processes from a perturbed configuration (achieved, for instance, via the application of a field pulse) towards a minimum in the energy landscape. ${ }^{3-9}$ However, the discovery of the possibility to modify the magnetic state of a nanomagnet by means of the spin-polarized current ${ }^{10}$ has opened a new route in the soliton dynamical studies and in their application in nanoscale microwave oscillators. ${ }^{11}$ In spin-valves or magnetic tunnel junctions, where the ground state of the ferromagnet was a magnetic vortex, several experiments showed the possibility to excite vortex selfoscillations. ${ }^{12,13}$ In different geometries, where the spinpolarized current was non-uniformly injected via a nanoaperture, the excitation of vortex-antivortex pairs ${ }^{14}$ and dissipative droplets ${ }^{15,16}$ was observed for in-plane and out-of plane magnetized ferromagnets, respectively.

In this paper, we present a systematic study of the dynamical properties of soliton modes excited in spin-valves which have a free layer with perpendicular to-sample-plane magnetic anisotropy (PMA). In detail, we study two different scenarios. In the first one, we are able to excite a soliton mode related to the rotation of two bubble/antibubble pairs localized near the nano-aperture with a sub-critical Hopf bifurcation at the critical current. The second scenario is in the framework of a very recent experimental study. ${ }^{16}$ Here, we find out that the topological density of the dissipative

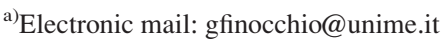

droplet can be related to a single constrained bubble/antibubble pair.

The approach used in this paper, based on the characterization of the dynamical properties of a soliton mode via the time domain variation of the topological density, can be also useful in the fundamental understanding of non-uniform magnetization dynamics and for a better design of soliton spin-torque nano-oscillators.

The paper is organized as follows. Section II reviews and introduces concepts of different magnetic bubble static states. In Sec. III, the micromagnetic formulation used for our numerical experiment is presented. Sections IV and V describe the results obtained in the two different frameworks. Section VI is devoted to further discussions and conclusions.

\section{OVERVIEW OF MAGNETIC BUBBLE STATES}

Let us consider a ferromagnetic thin film with strong perpendicular anisotropy and thus suppose that the ferromagnetic state is when all spins are pointing either up or down. When the spins are pointing opposite to the ferromagnetic state in a small circular area then we call this area magnetic bubble. Of course there is also a circular domain wall separating the bubble from the ferromagnetic state, and there are various possibilities for the domain wall (see, for example, Fig. 1 in Ref. 2).

In order to characterize the complexity of the bubble structure (also called the topological structure), it is useful to consider the following formula:

$$
\mathcal{N}=\frac{1}{4 \pi} \int n(x, y) d x d y,
$$

where $n=\mathbf{m} \cdot\left(\partial_{y} \mathbf{m} \times \partial_{x} \mathbf{m}\right)$ and $\mathbf{m}$ is the magnetization normalized to its saturation value $M_{S}$. The quantities $n$ and $\mathcal{N}$ are the topological density and the skyrmion number; ${ }^{1,2}$ this 
latter parameter is always an integer number for a bubble. The bubble with $\mathcal{N}=1$ is usually simply called a bubble while the one with $\mathcal{N}=-1$ an antibubble. Higher skyrmion numbers indicate higher complexity of the structure. ${ }^{2}$ The topological density $n=n(x, y)$ is defined at every point on the film. For the $\mathcal{N}=1$ bubble, $n$ has positive values at the domain wall and inside the bubble and it drops to zero outside it. For the $\mathcal{N}=-1$ antibubble, it simply has negative values around the wall and inside the bubble.

One interesting aspect to underline is that the magnetic domain which separates the reversed region related to the magnetic bubble from the rest of the ferromagnet could be not circular and the identification of the structure is difficult just by looking at them. In order to clarify the discussion, let us review some examples of various magnetic bubbles and bubble-antibubble pairs. Fig. 1 shows vector plots of the inplane magnetization $\left(m_{\mathrm{x}}, m_{\mathrm{y}}\right)$, and the corresponding (colorcoded) plots (red positive-blue negative) of the topological density $n(x, y)$ for different magnetic configurations. In all

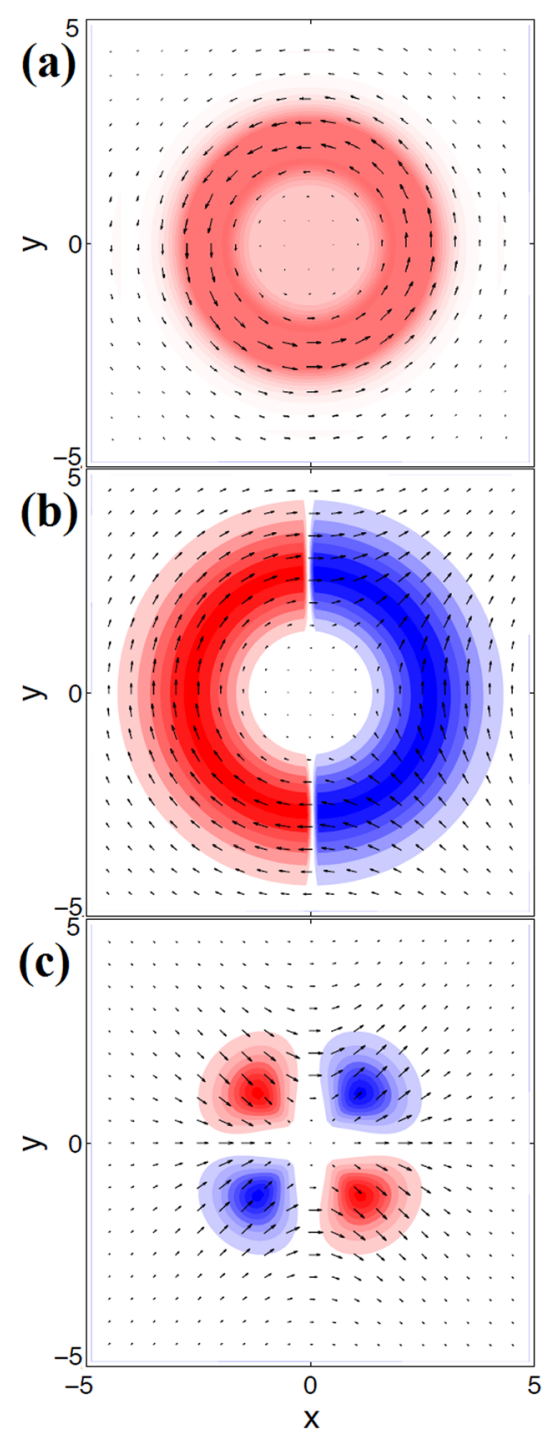

FIG. 1. Vector plot for $\left(m_{\mathrm{x}}, m_{\mathrm{y}}\right)$ and color-coded plot for the topological density $n$ (blue negative, red positive). (a) Magnetic bubble with $\mathcal{N}=1$. (b) Magnetic bubble with $\mathcal{N}=0$ called bubble-antibubble pair. (c) Magnetic bubble with $\mathcal{N}=0$ that can be viewed as two bubble-antibubble pairs. cases, the $m_{\mathrm{z}}$ component of the magnetization (not shown) is $m_{\mathrm{z}}=+1$ outside the bubble and $m_{\mathrm{z}}=-1$ inside the bubble (i.e., at roughly a circular region in the center of the figure).

In detail, Fig. 1(a) refers to a magnetic bubble with $\mathcal{N}=1$. The color-coded plot highlights that the topological density is mainly positive at the domain wall while it is zero elsewhere. Fig. 1(b) shows a magnetic bubble with $\mathcal{N}=0$. We see a region where $n>0$ and a region where $n<0$. For this reason, the structure may also be viewed as a bubbleantibubble pair. Lastly, Fig. 1(c) illustrates another structure with $\mathcal{N}=0$. In this case, given that we have two regions of positive and two regions of negative topological density, we can view the structure as two bubble-antibubble pairs.

For the sake of clarity, in the rest of the paper we refer to the topological structures described in Fig. 1 as "soliton modes" being our study addressed to the dynamical behavior of the "bubbles."

\section{MICROMAGNETIC MODELING}

As underlined above, in this numerical experiment, we study two different scenarios. In the first part of the paper, a point-contact geometry with both the free layer and the polarizer composed of perpendicular materials is investigated. In the last part, we refer to the same experimental device of Ref. 16 composed of a nano-contact, an in-plane polarizer and a perpendicular free layer. As discussed later in the text, the soliton modes excited in those systems are different being characterized by topological densities similar to the ones displayed in Figs. 1(c) and 1(b), respectively. In both cases, the dynamics of the magnetization vector of the FL, $\mathbf{M}=\mathbf{M}(t, \mathbf{r})$, is governed by the Landau-Lifshitz-GilbertSlonczewski (LLGS) equation ${ }^{10}$

$$
\frac{\partial \mathbf{M}}{\partial t}=\gamma\left[\mathbf{H}_{\mathrm{eff}} \times \mathbf{M}\right]+\frac{\alpha}{M_{\mathrm{S}}}\left[\mathbf{M} \times \frac{\partial \mathbf{M}}{\partial t}\right]+f\left(r / R_{\mathrm{c}}\right) \frac{\sigma I}{M_{\mathrm{S}}}[\mathbf{M} \times(\mathbf{M} \times \mathbf{p})],
$$

where the first term on the right-hand side represents the conservative precessional motion around the effective magnetic field $\mathbf{H}_{\text {eff }}$. Here, $\gamma$ is the gyromagnetic ratio and $\mathbf{H}_{\text {eff }}$ includes contributions from magnetostatic, exchange, Oersted, Zeeman, magnetostatic coupling, and magneto-crystalline anisotropy fields. The second term of Eq. (2) is the magnetic damping torque written in the traditional Gilbert form $(\alpha$ is the damping constant and $M_{\mathrm{S}}=|\mathbf{M}|$ is the saturation magnetization of the FL). The last term is the Slonczewski spintransfer torque that is proportional to the bias current $I$. The function $f\left(r / R_{\mathrm{c}}\right)$ describes the spatial distribution of the current across the cross section of the nanocontact. The coefficient $\sigma$ is related to the dimensionless spin polarization efficiency $\varepsilon$ by $\sigma=\varepsilon g \mu_{\mathrm{B}} / 2 e M_{S} S t_{\mathrm{FL}}$, where $g$ is the spectroscopic Landé factor, $\mu_{\mathrm{B}}$ is the Bohr magnetron, $e$ is the absolute value of the electron charge, $t_{\mathrm{FL}}$ is the FL thickness, and $S=\pi R_{\mathrm{c}}^{2}$ is the nanocontact area. The unit vector $\mathbf{p}$ defines the spin-polarization direction. It is computed by solving the Eq. (2) for the PL magnetization with $I=0$ and $\frac{\partial \mathbf{M}}{\partial t}=0$ $\left(\mathbf{p} \times \mathbf{H}_{\text {eff-p }}=0\right)$, the resulting $\mathbf{p}$ coincides with the equilibrium direction of the $\mathrm{PL}$ magnetization parallel to the 
effective field in the PL. For the dynamical behavior, the LLGS equation is numerically solved by using a 3-D dynamical code. ${ }^{17,18}$

\section{A. Spin-valve point-contact geometry}

The spin valve consists of a soft thin ferromagnetic layer and a thick hard layer, usually referred as free layer (FL) and pinned layer (PL), respectively, separated by a nonferromagnetic spacer. On the top of the FL, a metallic contact is realized to let the current flow through the structure. In detail, $\mathrm{PL}$ is $10 \mathrm{~nm}$ thick and made of $\mathrm{CoPt} / \mathrm{CoNi}$, the spacer is 10 nm-thick $\mathrm{Cu}$, and $\mathrm{FL}$ is CoNi. Its thickness, $t_{\mathrm{FL}}$, is a variable parameter of our analysis. The cross section of the spinvalve is circular with a diameter of $250 \mathrm{~nm}$. The contact is circular as well, centrally positioned on the top of the FL, with a diameter of $40 \mathrm{~nm}$ (see Fig. 2(a)). We used a 2D mesh of discretization cells having sizes $5 \times 5 \times t_{\mathrm{FL}} \mathrm{nm}^{3}$. The magnetic parameters used for the FL simulations are typical experimental values: saturation magnetization is $M_{\mathrm{S}}=650$ $\times 10^{3} \mathrm{~A} / \mathrm{m}$, exchange constant is $A=1.3 \times 10^{-11} \mathrm{~J} / \mathrm{m}$, and perpendicular magnetic anisotropy constant is $K_{\mathrm{u}}=3.3 \times 10^{5}$ $\mathrm{J} / \mathrm{m}^{3} .19$

\section{B. Continuous film nano-contact}

The continuous film nano-contact is composed of a metallic nanocontact with a radius of $30 \mathrm{~nm}$ on the top of a $\mathrm{Co} / \mathrm{Cu} / \mathrm{Co}-[\mathrm{Ni} / \mathrm{Co}]_{\mathrm{x} 4}$ orthogonal thin film with a squared section (see Fig. 2(b)). ${ }^{16}$ The FL is $4 \mathrm{~nm}$ thick and presents a perpendicular-to-plane anisotropy that is defined by the value of the anisotropy constant, $K_{\mathrm{u}}=3.0 \times 10^{5} \mathrm{~J} / \mathrm{m}^{3}$. The saturation magnetization is $M_{\mathrm{S}}=560 \times 10^{3} \mathrm{~A} / \mathrm{m}$, and the exchange constant is set at $A=1.3 \times 10^{-11} \mathrm{~J} / \mathrm{m}$. The metallic spacer is $4 \mathrm{~nm}$ in thickness. Lastly, the pinned layer is 12-nm-thick cobalt, with saturation magnetization $M_{\mathrm{SP}}=1500$ $\times 10^{3} \mathrm{~A} / \mathrm{m}$.

Differently from the previous system, an external magnetic field with a large amplitude of $1 \mathrm{~T}$ and inclination $75^{\circ}$ out-of-plane is applied to the system in order to tilt out of plane the magnetization of the in-plane polarizer (we estimated a tilted angle of $11^{\circ}$ ). In our numerical experiments, we restrict our study to a limited square computational region as large as $L \times L \times d_{F L}=500 \times 500 \times 4 \mathrm{~nm}^{3}$, by using a $2 \mathrm{D}$ mesh of discretization cells having sizes $2.5 \times 2.5 \times 4 \mathrm{~nm}^{3}$. As discussed in a previous work, ${ }^{20}$ to avoid the spurious spin-wave reflection from the computational boundaries we implement abrupt absorbing boundary conditions. Tests performed with $L \times L \times d_{F L}=750 \times$ $750 \times 4 \mathrm{~nm}^{3}$ gives rise to qualitatively same results with a quantitative difference in the oscillation frequency within $5 \%$.

\section{RESULTS: SPIN-VALVE POINT CONTACT GEOMETRY}

The first set of numerical simulations focus on the dynamics excited by the spin-polarized current with no external field. Then, we simulate the behavior of the device when subject to an in-plane external bias field of $50 \mathrm{mT}$, applied along the $x$ axis. All the simulations are performed starting from a parallel configuration of the magnetization.

\section{A. Zero-field dynamics}

A dissipative soliton mode is excited in the FL of the spin-valve when the applied current density is larger than a threshold value, $J_{\mathrm{ON}}$. We find that the oscillation amplitude at this critical current is finite, the oscillation axis is in the $\mathrm{z}$-direction, and it is reversed of $180^{\circ}$ with respect to the equilibrium configuration. Nonetheless, after it is excited, the soliton mode disappears for a smaller value of the current, $J_{\mathrm{OFF}}$. This additional presence of hysteresis in the excitation current is a clear indication that we are dealing with a subcritical Hopf-bifurcation. ${ }^{21}$ Such a clear hysteretic behavior is systematically studied by determining the values of $J_{\mathrm{ON}}$ and $J_{\mathrm{OFF}}$ for different values of the FL thickness. Results are summarized in Fig. 3(a). The thicker is the FL, the larger is the current density needed to excite the soliton mode. On the contrary the $J_{\mathrm{OFF}}$ exhibits a smoother increase with the FL thickness. The magnetic configuration, however, is not static, as it is shown in Fig. 3(b) where the average components of the magnetization under the contact area along the $x, y$, and $z$ directions are displayed, corresponding
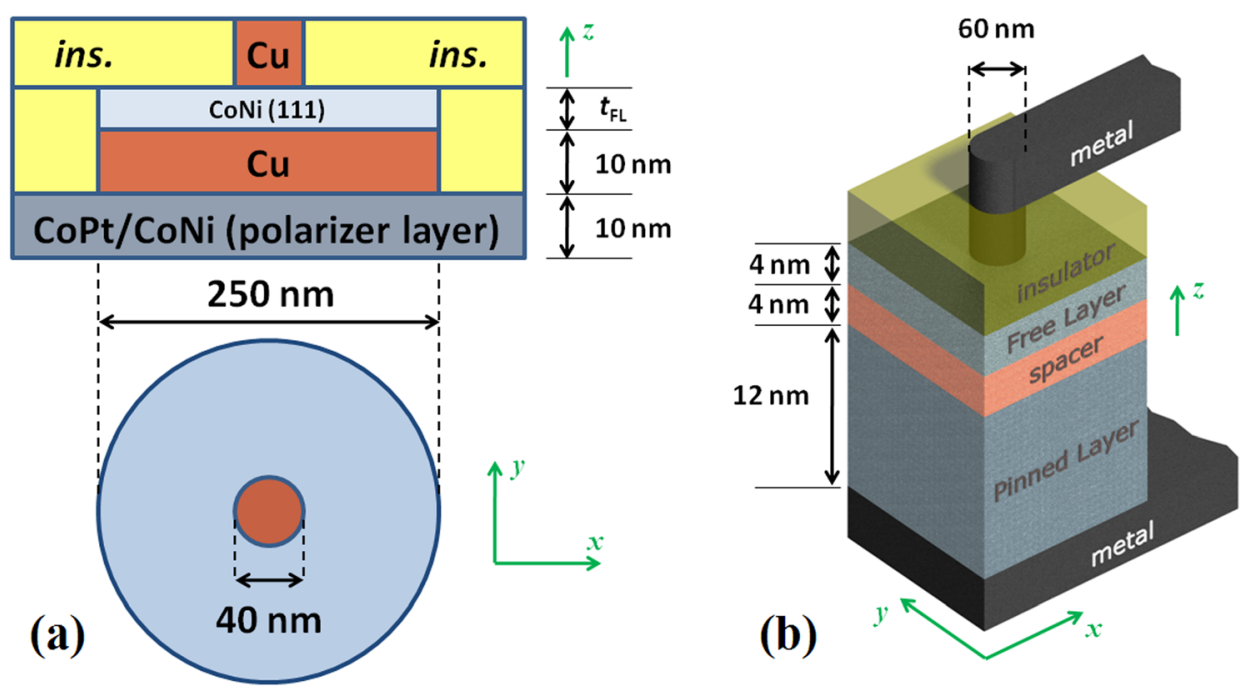

FIG. 2. (a) Schematic of the spin-valve point-contact geometry. The spin-valve has a circular cross section of $250 \mathrm{~nm}$ of diameter, while the diameter of the $\mathrm{Cu}$ contact is $40 \mathrm{~nm}$. (b) Sketch of the continuous film nano-contact spin-torque oscillator. The nanocontact has a diameter of $60 \mathrm{~nm}$. 

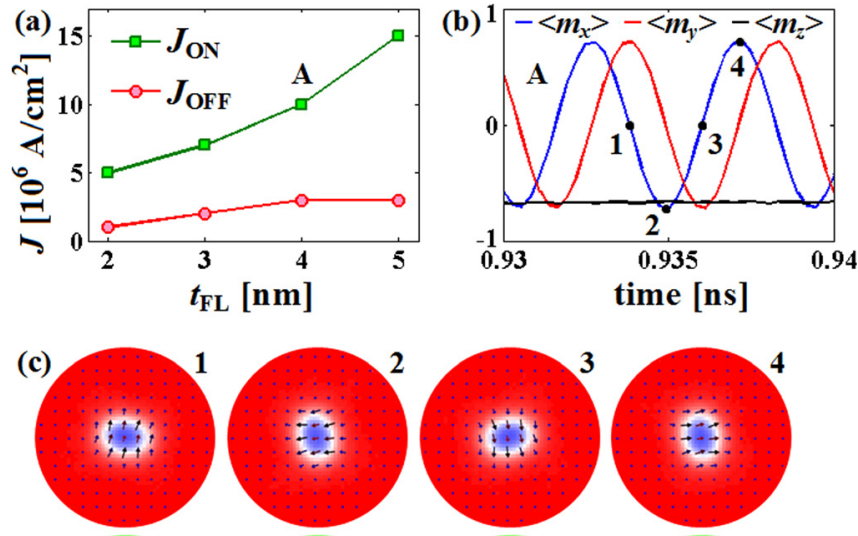

(d)

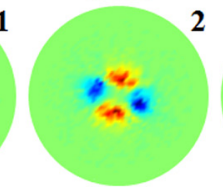

2
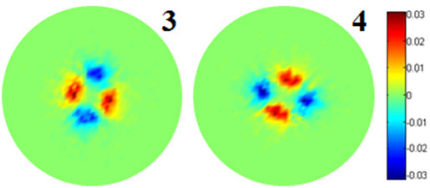

FIG. 3. Zero-field dynamics. (a) Threshold currents vs. FL thickness. The point "A" $\left(t_{\mathrm{FL}}=4 \mathrm{~nm}, J=J_{\mathrm{ON}}=10 \times 10^{6} \mathrm{~A} / \mathrm{cm}^{2}\right)$ specifies the condition for the Figs. (b)-(d). (b) Average components of the magnetization under the contact area along the $x, y$, and $z$ directions, corresponding to the condition "A." (c) Snapshots of the magnetic configuration at four different instants of the dynamics 1-4 as indicated in Fig. 3(b). Red and blue colors indicate positive and negative $z$-component of the magnetization, respectively. (d) Snapshots of the topological density for the same points $1-4$ as in Fig. 3(c). Red and blue colors indicate positive and negative topological densities, respectively. This dynamical results are related to the device depicted in Fig. 2(a) (enhanced online) [URL: http://dx.doi.org/10.1063/1.4827384.1].

to the point "A" of Fig. 3(a) $\left(J=1 \times 10^{7} \mathrm{~A} / \mathrm{cm}^{2}\right.$ and $\left.t_{\mathrm{FL}}=4 \mathrm{~nm}\right)$. In detail, each spin precesses around its equilibrium position keeping its $z$ component constant. This happens both inside the reversed domain, where the magnetization precesses around the -1 position, and at its wall, where the magnetization rotates lying in the plane. Fig. 3(c) shows, as example, four snapshots of such a dynamical magnetic state related to the point 1-4 indicated in Fig. 3(b). As can be noted, the area of the reversed domain does not change significantly in time domain for a fixed current value.

In order to better describe the achieved magnetic configuration, we calculate the topological density over the FL as a function of time. The result indicates that there are two regions where $n$ is positive and two more regions where it is negative (Fig. 3(d)). We can say that two bubble-antibubble pairs are nucleated (compare Fig. 3(d) with Fig. 1(c)). In addition, the topological density configuration rotates counterclockwise as well as the magnetic configuration does (compare the four images in Fig. 3(d) to the ones in Fig. 3(c)).

The behavior of this magnetic soliton, as a function of the applied current, is summarized in Fig. $4\left(t_{\mathrm{FL}}=4 \mathrm{~nm}\right)$. In particular, our simulations show that an increase of the current flowing through the structure leads to a decrease of the output frequency ${ }^{15}$ and an increase of the area of the reversed domain. Qualitatively, the frequency reduction can be understood by considering that the time for a complete rotation of the topological density (strictly related to the output frequency) around the nano aperture is longer if the area of the reversed domain is larger.

The main experimental problem corresponding to this numerical framework is the measurement of the giant-

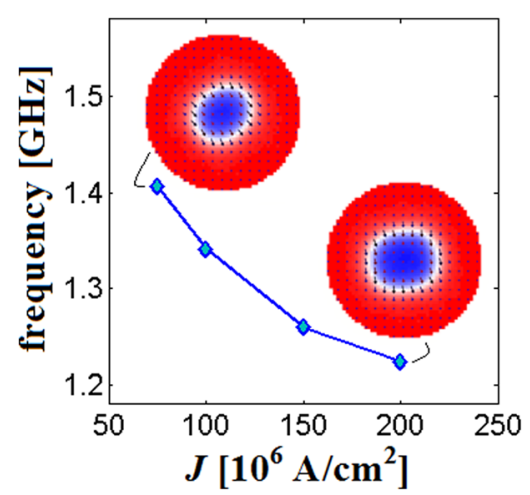

FIG. 4. Zero-field dynamics. Output frequency vs. applied current density for $t_{\mathrm{FL}}=4 \mathrm{~nm}$. The two insets represent the magnetic configurations (red and blue positive and negative out of plane component of the magnetization, respectively) corresponding to the terminal points of the analyzed range. As it is possible to see, the higher is the current the larger is the reversed domain area.

magnetoresistive (GMR) signal which comes from the $z$ component variation of the magnetization that here is small at zero field (as can be seen for example in Fig. 3(b) black line). For this reason, we have performed a similar analysis in the presence of an external in-plane field.

\section{B. In-plane field dynamics}

For an external magnetic field applied along the $\mathrm{x}$-direction, we systematically computed the oscillation frequency as a function of the applied current density for three different values of the FL thickness. Fig. 5(a) shows that the frequency decreases with increasing current, similarly to what happened without external field. In addition, for a fixed value of the current, the thicker the FL, the higher is the output frequency. This aspect can be qualitative explained by the reduction of the out-of-plane demagnetizing field at larger thickness.
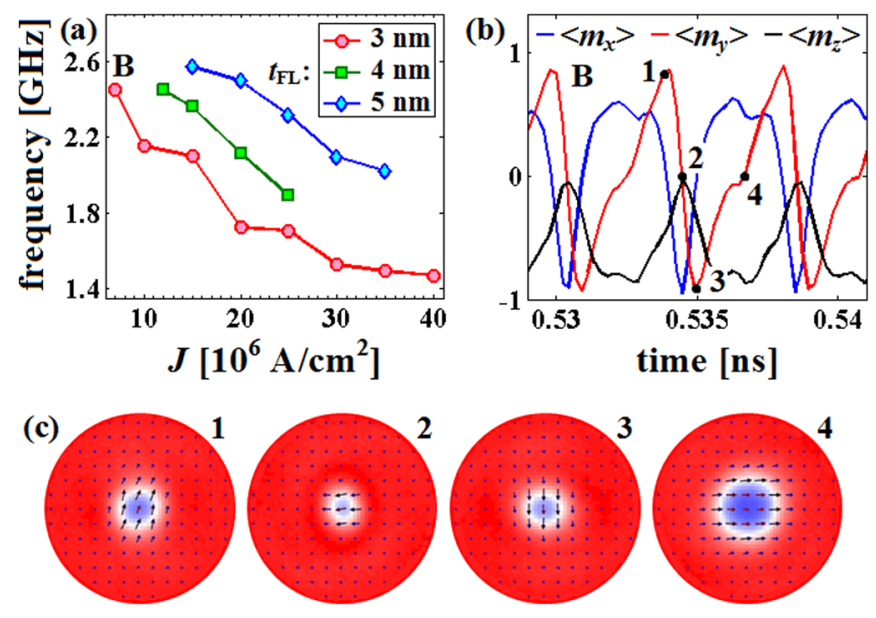

FIG. 5. In-plane field dynamics. (a) Output frequency vs. applied current density for three values of the FL thickness. The first value of current represents the switching off current for each value of FL thickness. The point "B" $\left(t_{\mathrm{FL}}=3 \mathrm{~nm}, J=J_{\mathrm{OFF}}=7 \times 10^{6} \mathrm{~A} / \mathrm{cm}^{2}\right)$ defines the condition for the Figs. (b) and (c). (b) Average components of the magnetization under the contact area along the $x, y$, and $z$ directions, corresponding to the condition "B." (c) Snapshots of the magnetic configuration at four different instants of the dynamics as indicated by the points $1-4$ in Fig. (b). Red and blue colors indicate positive and negative $z$-component of the magnetization, respectively (enhanced online) [URL: http://dx.doi.org/10.1063/1.4827384.2]. 
Fig. 5(b) shows the magnetization dynamics along the $x$, $y$, and $z$ directions for the point " $\mathrm{B}$ " $\left(J=7 \times 10^{6} \mathrm{~A} / \mathrm{cm}^{2}\right.$ and $\left.t_{\mathrm{FL}}=3 \mathrm{~nm}\right)$ as indicated in Fig. 5(a). As can be noted (Fig. 5(b) black line), the in-plane external magnetic field of $50 \mathrm{mT}$ is sufficient to give rise to a measurable oscillation of the $z$-component of the magnetization below the contact area which correspond to half of the maximum GMR signal achievable in those systems, therefore to the possibility of experimentally measuring the GMR signal.

The external field also modifies the structure of the excited mode. Actually, together with a rotational motion similar to the one obtained at zero field, a phenomenon of expansion and contraction of the reversed domain area is present. Moreover, it is possible to notice that the soliton mode enlarges while the in-plane component of the magnetization aligns with the field direction and shrinks while it goes to the opposite direction. Fig. 5(d) aims to show this feature by means of four different magnetization snap-shots.

\section{RESULTS: CONTINUOUS FILM NANO-CONTACT}

The numerical results obtained for the nano-contact geometry described at the point $B$ of Sec. III are shown in the left column of Figs. 6 and 7. The frequency of the excited dynamics is about $30 \mathrm{GHz}$ and slightly decreases with the
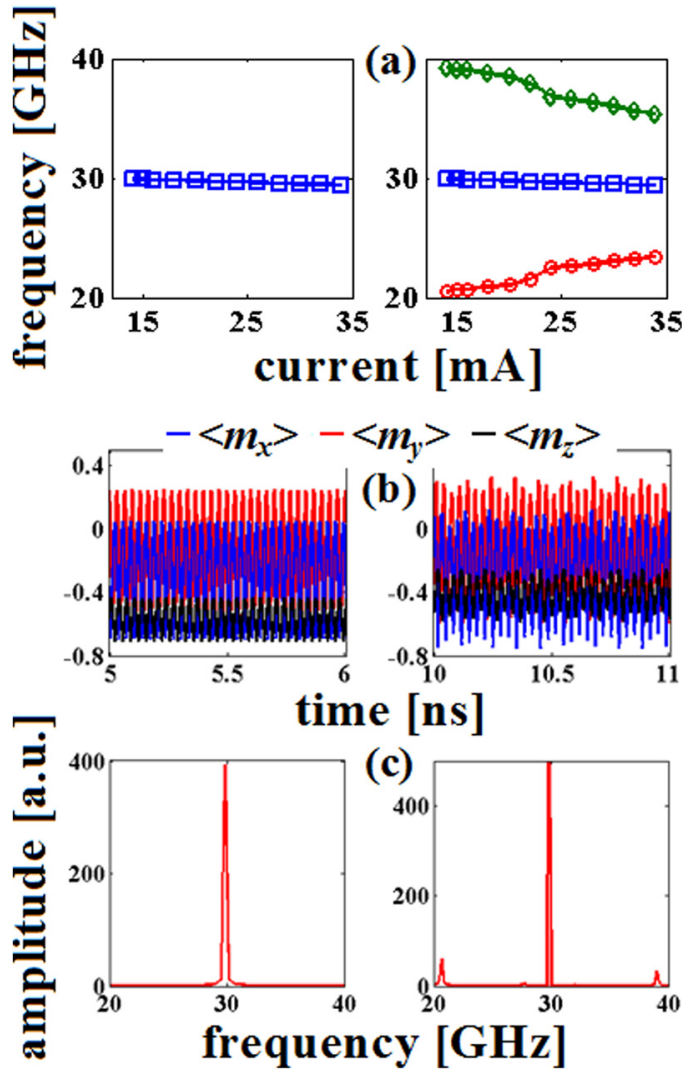

FIG. 6. Magnetization dynamics excited in an STNO for (left-column) $M_{\mathrm{S}}=560 \times 10^{3} \mathrm{~A} / \mathrm{m}$ and (right-column) $M_{\mathrm{S}}=510 \times 10^{3} \mathrm{~A} / \mathrm{m}$, the latter case corresponding to a self-modulation phenomenon. (a) Output frequencies versus applied current. The main frequency decreases with the current. In the case of self-modulation, two sidebands appear symmetrically with respect to the central peak. (b) Average components of the magnetization under the contact area along $\mathrm{x}, \mathrm{y}$, and $\mathrm{z}$. (c) Output spectrum. The right-column spectrum shows the same main peak of the left-column one plus two sidebands. applied current (Fig. 6(a)). Figs. 6(b) and 6(c) illustrate the average components of the magnetization under the contact area and the output spectrum, respectively, for an applied current of $I=16 \mathrm{~mA}$, corresponding to a current density of $J=5.85 \times 10^{8} \mathrm{~A} / \mathrm{cm}^{2}$.

The configuration of the magnetization is depicted in Fig. 7(a), where a clear soliton structure appears. Once again, the magnetization does not reach the vertical position -1 , but it precesses around it. The calculus of the topological density over the film, shown in Fig. 7(b), highlights that the excited structure is a bubble-antibubble pair (see Fig. 1(b) for comparison). Such results confirm the experimental achievements; ${ }^{16}$ nonetheless, we can say that the droplets observed in those experiments have, in this case, the structure of a partially developed bubble-antibubble. The key ingredient which is different from the previous numerical experiments is the presence of a large in-plane component (around $260 \mathrm{mT}$ ), which brings the symmetry of the system to favor the excitation of a bubble/antibubble pair instead of two couples of bubble/antibubble.

With the aim of finding dynamics with different features, we performed a systematic study by considering small variation of the main parameters of the structure. The most interesting achievements are obtained by decreasing the saturation magnetization of the FL. In these cases, in fact, a wide current range of self-modulation phenomenon is observed as well as it happened in the experiments. ${ }^{16}$ The right column of Figs. 6 and 7 summarize the numerical results for $M_{\mathrm{S}}=510 \times 10^{3} \mathrm{~A} / \mathrm{m}$.

In detail, by varying the applied current, we get a main frequency that does not change with respect to the previous case. Nonetheless, here, we can also see two sidebands that get closer to the central peak while the current is increased

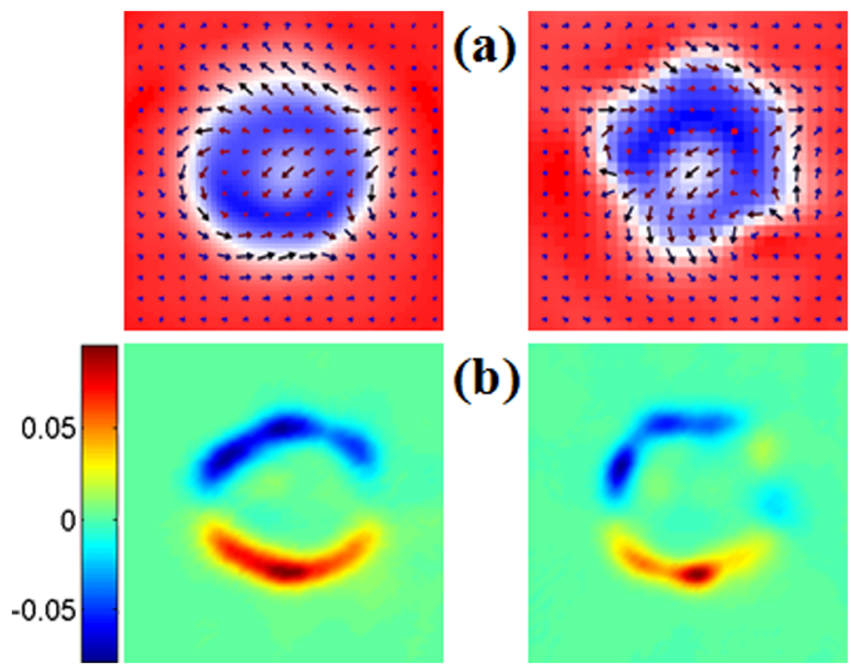

FIG. 7. Magnetization dynamics excited in an STNO for (left-column) $M_{\mathrm{S}}=560 \times 10^{3} \mathrm{~A} / \mathrm{m}$ and (right-column) $M_{\mathrm{S}}=510 \times 10^{3} \mathrm{~A} / \mathrm{m}$, the latter case corresponding to a self-modulation phenomenon. (a) Example of snapshots of the magnetic configuration. In the case of self-modulation, the bubble appears deformed. (b) Snapshots of the topological density distribution. The left-side image highlights the nucleation of a bubble-antibubble pair. On the right, apart from the two main regions that indicate a bubble-antibubble pair, it is possible to see two additional small regions due to the modulating signal (enhanced online) [URL: http://dx.doi.org/10.1063/1.4827384.3] [URL: http://dx.doi.org/10.1063/1.4827384.4]. 
(see Fig. 6(a)-right). The three components of the magnetization vary with a sinusoidal hull and the spectrum shows two bands at the sides of the main peak (see Figs. 6(b) and 6(c) that are obtained for $I=16 \mathrm{~mA}$, corresponding to a current density of $\left.J=5.85 \times 10^{8} \mathrm{~A} / \mathrm{cm}^{2}\right)$. The frequency distance between the main peak (still located at $30 \mathrm{GHz}$ and representing the carrier signal) and the sidebands is about $9 \mathrm{GHz}$, which can be seen as the frequency of the modulating wave.

The snapshot of the magnetic configuration of the FL (Fig. 7(a)-right) shows a soliton mode where the reversed domain is separated by a non-circular domain wall. The presence of this distortion can be one of the sources of selfmodulation phenomenon experimental found. Such an effect arises also in the topological density configuration. For example, Fig. 7(b) shows the two main regions corresponding to the main bubble-antibubble pair and two smaller regions corresponding to a pair of bubble-antibubble that acts as modulating quantity.

The differences between the two cases represented by the left and the right columns of Figs. 6 and 7 can be figured out by considering that for a fixed current density a decrease of the FL saturation magnetization leads to an increase of the nonlinearities in the system and, therefore, to more complicated excited modes.

\section{DISCUSSIONS AND CONCLUSIONS}

The numerical results described in the previous two sections merit further discussions. First of all, one could argue that the simple magnetic configuration in Fig. 1(a) $(\mathcal{N}=1)$ has the same vector structure as produced by the Oersted field. We have performed several simulations without and with Oersted field, and in all the cases the strength of this field is not enough to excite a soliton mode with the topological density as depicted in Fig. 1(a). In other words, in these kind of structures, the dynamical response does not depend qualitatively on the Oersted field.

In both systems, after the current density is applied the stationary magnetization precession is reached from the uniform configuration in a short transient regime $(<15 \mathrm{~ns})$. In particular, in spin-valve nanopillars, the transient regime is strongly non-linear with the excitation of incoherent spinwaves. ${ }^{22}$ Contrarily, for the continuous film case, the transient is characterized by a magnetization precession around the equilibrium configuration (coherent spin-waves), which increases up to the reversing of the magnetization oscillation below the nano-aperture. ${ }^{22}$ This difference is related to the dimension of the free layer cross section: while in the first system the effects of the spatial confinement are important (the transient spin-waves propagate back and forth), in the second case they are negligible (the transient spin-waves propagate far from the nano-aperture with no reflection).

One more issue is that the obtained configurations are rather similar to the droplets shown in Refs. 1 and 15. In the former work, in fact, pairs of bubble/antibubbles propagating in the structure were studied. We have to note that our framework include a damping effect that is absent in the study of Ref. 1. Basically, the damping losses are compensated only in the region where the current is injected, this gives rise to a restore damping force which maintains the soliton mode localized near that region.

In the scenario of Ref. 15, the droplets move away from the area where the current is injected after its nucleation, the movement is driven by the presence of an external magnetic field gradient. In our systems, no field gradient is applied, and in addition, after the current is switched off, the solitons are not stable and they rapidly disappear.

Finally, we compare our results with the computations of Refs. 15 and 16. The dissipative droplet shown in Fig. 3 of Ref. 15 is obtained in the high simmetry case, i.e. when both free and fixed layers are perpendicular. It presents all vectors pointing to the same in-plane direction and the topological density $n$ is zero everywhere. The scenario changes in Fig. 6 of Ref. 15, where the Oersted field is taken into account and both the external field and the polarizer are nearly perpendicular to plane (as in our numerical framework). Here, the droplet seems to consist of half bubble and half antibubble in a way similar to our sketch in Fig. 1(b) and we can infer that the two regions are characterized by a topological density $n$ with different sign. In conclusion, the results of the micromagnetic simulations described in Sec. V are qualitatively the same to the results of Fig. 6 of Ref. 15 and Fig. 3(a) of Ref. 16; however, here we classify the dissipative droplet in term of its topological density as bubble/antibubble pair.

From a general point of view, our results are important in the theoretical understanding on how to control the spatial structure of soliton modes for application in spintronics, magnonics, and domain wall devices.

\section{ACKNOWLEDGMENTS}

The authors thank Alessandro Prattella for his support in the numerical simulations. This work was supported by project PRIN2010ECA8P3 from Italian MIUR, project MAT2011-28532-C03-01 from Spanish government, and project SA163A12 from Junta de Castilla y Leon.

\footnotetext{
${ }^{1}$ A. M. Kosevich, B. A. Ivanov, and A. S. Kovalev, Phys. Rep. 194, $117-238$ (1990)

${ }^{2}$ N. Papanicolaou and T. N. Tomaras, Nucl. Phys. B 360, 425 (1991).

${ }^{3}$ N. R. Cooper, Phys. Rev. Lett. 80, 4554-4557 (1998).

${ }^{4}$ B. A. Ivanov and A. M. Kosevich, Zh. Eksp. Teor. Fiz. 72, 2000 (1977).

${ }^{5}$ V. L. Pokrovskii and G. V. Uimin, Sov. Phys. JETP 41, 128 (1985).

${ }^{6}$ A. M. Kosevich, V. V. Gann, A. I. Zhukov, and V. P. Voronov, Zh. Éksp. Teor. Fiz. 114, 735-746 (1998).

${ }^{7}$ S. Komineas, Phys. Rev. Lett. 99, 117202 (2007).

${ }^{8}$ C. Moutafis, S. Komineas, C. A. F. Vaz, J. A. C. Bland, T. Shima, T. Seki, and K. Takanashi, Phys. Rev. B 76, 104426 (2007).

${ }^{9}$ D. Urzagasti, D. Laroze, M. G. Clerc, S. Coulibaly, and H. Pleiner, J. Appl. Phys. 111, 07D111 (2012).

${ }^{10}$ J. Slonczewski, J. Magn. Magn. Mater. 159, L1 (1996).

${ }^{11}$ Z. Zeng, G. Finocchio, and H. Jiang, Nanoscale 5, 2219 (2013).

${ }^{12}$ G. Finocchio, V. S. Pribiag, L. Torres, R. A. Buhrman, and B. Azzerboni, App. Phys. Lett. 96, 102508 (2010).

${ }^{13}$ A. Dussaux, B. Georges, J. Grollier, V. Cros, A. V. Khvalkovskiy, A. Fukushima, M. Konoto, H. Kubota, K. Yakushiji, S. Yuasa, K. A. Zvezdin, K. Ando, and A. Fert, Nat. Commun. 1, 8 (2010).

${ }^{14}$ G. Finocchio, O. Ozatay, L. Torres, R. A. Buhrman, D. C. Ralph, and B. Azzerboni, Phys. Rev. B 78, 174408 (2008).

${ }^{15}$ M. A. Hoefer, T. J. Silva, and M. W. Keller, Phys. Rev. B 82, 054432 (2010).
} 
${ }^{16}$ S. M. Mohseni, S. R. Sani, J. Persson, T. N. Anh Nguyen, S. Chung, Ye. Pogoryelov, P. K. Muduli, E. Iacocca, A. Eklund, R. K. Dumas, S. Bonetti, A. Deac, M. A. Hoefer, and J. Akerman, Science 339, 1295 (2013).

${ }^{17}$ G. Finocchio, A. Prattella, G. Consolo, E. Martinez, A. Giordano, and B. Azzerboni, J. Appl. Phys. 110, 123913 (2011).

${ }^{18}$ A. Giordano, G. Finocchio, L. Torres, M. Carpentieri, and B. Azzerboni, J. Appl. Phys. 111, 07D112 (2012); M. Carpentieri, E. Martinez, and G. Finocchio, ibid. 110, 093911 (2011).

${ }^{19}$ T. Hauet, E. Dobisz, S. Florez, J. Park, B. Lengsfield, B. D. Terris, and O. Hellwig, Appl. Phys. Lett. 95, 262504 (2009).
${ }^{20}$ V. Puliafito, B. Azzerboni, G. Consolo, G. Finocchio, L. Torres, and L. Lopez-Diaz, IEEE Trans. Magn. 44, 2512 (2008); M. Carpentieri, T. Moriyama, B. Azzerboni, and G. Finocchio, Appl. Phys. Lett. 102, 102413 (2013).

${ }^{21}$ Y. A. Kuznetsov, Elements of Applied Bifurcation Theory, 2nd ed. (Springer, 1998).

${ }^{22}$ See supplementary material at http://dx.doi.org/10.1063/1.4827384 for transient time evolution of the spatial distribution of the magnetization for the case of spin-valve at zero field and for transient time evolution of the spatial distribution of the magnetization for the case of continuous film. 